\title{
Low-energy Spectra of the $t$-J-Type Models Studied by Variational Approach
}

\author{
Chung-Pin $\mathrm{Chou}^{1,2}$, T. K. Lee ${ }^{2}$, and Chang-Ming $\mathrm{Ho}^{3}$ \\ ${ }^{1}$ Department of Physics, National Tsinghua University, Hsinchu 300, Taiwan \\ ${ }^{2}$ Institute of Physics, Academia Sinica, Nankang 115, Taiwan \\ ${ }^{3}$ Department of Physics, Tamkang University, Taipei 251, Taiwan
}

(Dated: November 29, 2018)

\begin{abstract}
We discuss recent progress of understanding the phenomena observed in high $T_{c}$ cuprates by studying the $d$-wave resonating-valence-bond ( $d$-RVB) based trial wave functions for the 2-dimensional $t$ - $J$-type models. Treat exactly the strong correlation between electrons by numerical approach, we examine the evolution of ground states described by different variational wave functions and properties of the quasi-hole and -particle excitations of the $d$-RVB superconducting (SC) state. Properties related to the Fermi surface geometry deduced from quasi-hole energy dispersion of the SC state is shown to be consistent with the observation by photoemission spectroscopy. With the calculated spectral weights (SW's) for adding and removing an electron, we found not only the anti-correlation between conductance peak height and width between peaks seen in tunneling experiments, but also unique properties due to strong correlation which need to be verified by future experiments.
\end{abstract}

The emergence of superconductivity as holes or electrons doped into the insulating parent compounds is one of the fascinating facts seen in high $T_{c}$ cuprates ${ }^{1}$. In addition to that, there are many other intriguing phenomena observed in the underdoped regime which may indicate new and even exotic phases $\stackrel{1}{\text {. }}$. Phases at different doping levels have been characterized experimentally by studying in detail the low-energy, single-particle spectra by applying, for example, angle-resolved photoemission spectroscopy (ARPES) and scanning tunneling microscopy/spectroscopy (STM/STS) 2,3,4,5,6.

To understand these phenomena, we have been studying the trial wave functions (TWF's), in the form of the resonating-valence-bond (RVB) state proposed by Anderson ${ }^{7}$, for the $t$ - $J$-type model with different concentration of doped holes or electrons. Some recent progress of this work is discussed in this paper. We start by discussing the different variational WF's describing ground states at different doping levels. Although our study is for only the uniform, 2-dimensional case representing the ideal $\mathrm{CuO}$ plane without any disorders (due to lattice, inhomogeneous doping etc.), the phases we found, in particular, the antiferromagnetic metal $(\mathrm{AFMM})^{8}$ and the coexisting state of superconductivity and antiferromagnetism (AFMM+SC) ${ }^{9}$ (see Fig 1 below)- have recently been observed, for the first time, in the $\mathrm{Hg}$ based 5-layer cuprates 10 .

We have also extended the TWF approach to examine properties of the low-energy excitation of the $d$-wave RVB ( $d$-RVB) state in the superconducting regime. In the second part of this paper, we present the doping dependence of Fermi surfaces, extracted from the quasi-hole energy dispersion, is consistent with results obtained by ARPES. Furthermore, we have calculated the spectral weights (SW's) of the quasi-hole and -particle excitations and made comparison of the quantities derived from them with the observation made by STM/STS.

Evolution of ground states with doping. Let us first discuss the TWF's for ground states of the $t$ - $J$-type Hamiltonian $\boldsymbol{H}=\sum_{i, j \sigma}-t_{i j} \tilde{c}_{i \sigma}^{\dagger} \tilde{c}_{j \sigma}+$ H.c. $+\boldsymbol{H}_{J}$ with dif- ferent numbers of holes or electrons. Here $i, j$ in the summation represents nearest-neighbor (n.n.) sites the electron hops (with amplitude $t$ ), the second n.n. $\left(t^{\prime}\right)$, third n.n. site $\left(t^{\prime \prime}\right)$ pairs and the Heisenberg exchange term $\boldsymbol{H}_{J}=J \sum_{\langle i, j\rangle}\left(\mathbf{S}_{i} \cdot \mathbf{S}_{j}-\frac{1}{4} n_{i} n_{j}\right)$ between n.n. electrons. Note that $\tilde{c}_{i \sigma}$ in $\boldsymbol{H}$ creates different kinds of holes from single-electron-occupied sites: empty holes (0e-hole) for hole doping and two-electron-occupied holes (2e-hole) for electron doping ${ }^{11}$. Namely, $\tilde{c}_{i \sigma}$ is actually equal to $c_{i \sigma}\left(1-n_{i,-\sigma}\right)$ or $c_{i,-\sigma} n_{i \sigma}$ for hole or electron doped case, respectively.

Taking the point of view of doping the Mott insulator with antiferromagnetic long range order (AF LRO), we discuss TWF's determined variationally at different doping levels from the undoped case. The TWF's we constructed may include three mean-field parameters, depending on the doping concentration: the staggered magnetization $m_{s}=\left\langle S_{A}^{z}\right\rangle=-\left\langle S_{B}^{z}\right\rangle$, where the lattice is divided into two sublattices when AF LRO is present; the uniform bond order parameters $\chi=\left\langle\sum_{\sigma} c_{i \sigma}^{\dagger} c_{j \sigma}\right\rangle$; and $d$-RVB order $\Delta=\left\langle c_{j \downarrow} c_{i \uparrow}-c_{j \uparrow} c_{i \downarrow}\right\rangle$ if $i$ and $j$ are n.n. sites in $x$ direction and $-\Delta$ in $y$-direction.

The TWF for lightly doped case is a generalization of the single-hole WF with explicit AF LRO first written down by Lee and Shih ${ }^{12}$. In contrast to states at higher doping (see below), WF in this regime is constructed solely from the optimized one at half-filling, i.e. with Hamiltonian $\boldsymbol{H}_{J}$,

$$
\left|\Psi_{0}\right\rangle=P_{d}\left[\sum_{\mathbf{k}[\mathbf{S B Z}]}\left(A_{\mathbf{k}} a_{\mathbf{k} \uparrow}^{\dagger} a_{-\mathbf{k} \downarrow}^{\dagger}+B_{\mathbf{k}} b_{\mathbf{k} \uparrow}^{\dagger} b_{-\mathbf{k} \downarrow}^{\dagger}\right)\right]^{N_{s} / 2}|0\rangle .
$$

Here coefficients $A(B)_{\mathbf{k}}=\left[+(-) E_{\mathbf{k}}+\xi_{\mathbf{k}}\right] / \Delta_{\mathbf{k}}$ with $E_{\mathbf{k}}=\left(\xi_{\mathbf{k}, 0}^{2}+\Delta_{\mathbf{k}}^{2}\right)^{1 / 2} . \quad \xi_{\mathbf{k}, 0}=\left[\epsilon_{\mathbf{k}}^{2}+\left(m_{s v}\right)^{2}\right]^{\frac{1}{2}}$ are energy dispersions for the two spin density wave (SDW) bands with $\epsilon_{\mathbf{k}}=-\left(\cos \mathrm{k}_{x}+\cos \mathrm{k}_{y}\right) . \quad a_{\mathbf{k} \sigma}$ and $b_{\mathbf{k} \sigma}$ represent the operators of the lower and upper SDW bands, respectively, and are related to the original electron operators $c_{\mathbf{k} \sigma}$ and $c_{\mathbf{k}+\mathbf{Q} \sigma}$ with $\mathbf{Q}=(\pi, \pi)$ set for the commensurate SDW state. $\Delta_{\mathbf{k}}=\Delta_{v}\left(\cos k_{x}-\cos k_{y}\right)$ for the 
$d$-RVB order parameter. The projection operator $P_{d}$ enforces the constraint of one electron per site. At half-filling, $N_{s^{-}}$number of lattice sites- equals the total number of electrons. Notice that the sum in $\left|\Psi_{0}\right\rangle$ is taken over sublattice BZ (SBZ). There are two variational parameters: $\Delta_{v}$ and $m_{s v}$ in this WF. It has been shown that this WF produces both the staggered magnetization, $\langle m\rangle=N_{s}^{-1} \sum_{i}(-1)^{i} S_{i}^{z}$, and optimized energy very similar to those obtained by quantum Monte Carlo approach 13 .

When a hole is doped or an electron is removed from the Mott "vacuum" $\left|\Psi_{0}\right\rangle$, a pair must be broken with an unpaired spin left. Thus it is quite natural to have the following Lee-Shih WF for a single doped hole and a lone up spin, for example,

$$
\begin{aligned}
\left|\Psi_{1}(\mathbf{q})\right\rangle= & P_{d} c_{\mathbf{q} \uparrow}^{\dagger} \\
& {\left[\sum_{[\mathbf{k} \neq \mathbf{q}]}\left(A_{\mathbf{k}} a_{\mathbf{k} \uparrow}^{\dagger} a_{-\mathbf{k} \downarrow}^{\dagger}+B_{\mathbf{k}} b_{\mathbf{k} \uparrow}^{\dagger} b_{-\mathbf{k} \downarrow}^{\dagger}\right)\right]^{\left(N_{s} / 2\right)-1}|0\rangle, }
\end{aligned}
$$

where the hole momentum $\mathbf{q}$ is excluded from the sum if $\mathbf{q}$ is within the SBZ, otherwise, $\mathbf{q}-\mathbf{Q}$ is excluded. Note that this WF does not contain any information about hoppings of the doped hole or electron. Nevertheless, the effect of n.n. hopping is included in the RVB uniform bond which describes the large quantum fluctuation and spin singlet formation. There is also no need to introduce $t^{\prime}$ and $t^{\prime \prime}$ in the TWF as they are compatible with AF LRO. Variational energies calculated vary with $\mathbf{q}$ and we may obtain the energy dispersion for the $t-t^{\prime}-t^{\prime \prime}-J$ mode ${ }^{8.12}$. The ground state with one hole is found to have momentum $(\pi / 2, \pi / 2) \equiv \mathbf{Q} / 2$. As shown in Ref. $\frac{8}{}$, this dispersion relation is still followed when the hole number is increased. It is interesting to note that states in the hole- and electron-doped cases are in oneto-one correspondence after a local particle-hole transformation $c_{i \sigma} \rightarrow c_{i,-\sigma}^{\dagger}$ is made. Also, because of the Fermi statistics, the exchange of a single spin with a $2 e$-hole has an extra minus sign as compared to the $0 e$-hole. Hence, the only difference between the hole and electron doped $t-t^{\prime}-t^{\prime \prime}-J$ models is $t^{\prime} / t \rightarrow-t^{\prime} / t$ and $t^{\prime \prime} / t \rightarrow-t^{\prime \prime} / t$ after we change the $c_{i \sigma}$ on one sublattice sites to $-c_{i \sigma} 14$. With all these, we treat the hole and electron doped cases in the same manner. As for the case of having an extra up-spin electron with momentum q doped into the halffilled state, the energy dispersion can be calculated with this same $\left|\Psi_{1}\right\rangle$. The ground state is here at momentum $(\pi, 0)$. This result agrees well with that obtained by self-consistent Born approximation (see the discussion in Ref ${ }^{8}$ ). Dispersions for single-hole and -electron cases turn out to be simply the combination of the mean-field band at half-filling and the coherent hoppings 8.12. Using $\left|\Psi_{1}\right\rangle$, we calculated the momentum distribution function (MDF) $n_{\mathbf{k}}=\left\langle c_{\mathbf{k} \sigma}^{\dagger} c_{\mathbf{k} \sigma}\right\rangle$ for the ground state of, say, a single 0 -hole with momentum $\mathbf{Q} / 2$. We observed the same dips or pockets at $\mathbf{Q} / 2$ and anti-dips at $-\mathbf{Q} / 2$ found for the exact results of 32 sites (see Ref ${ }^{8}$ ).

We may generalize the WF to describe states with two

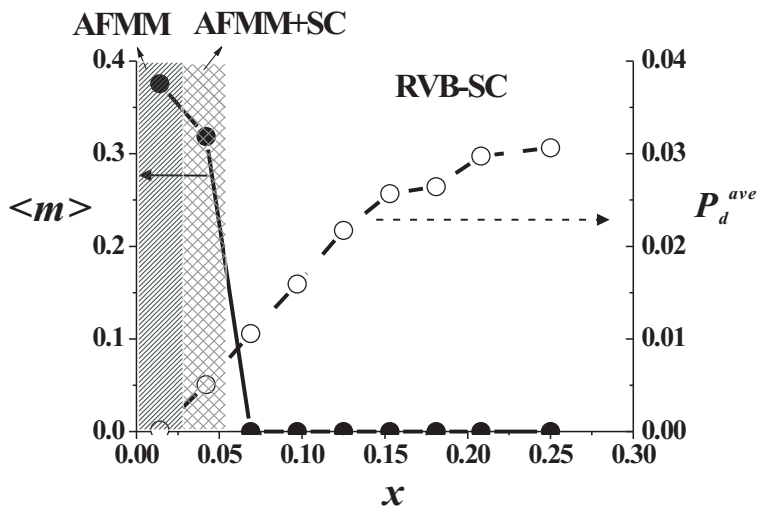

FIG. 1: Ground state phase diagram for the $t-t^{\prime}-t^{\prime \prime}-J$ model with $\left(t^{\prime}, t^{\prime \prime}\right) / t=(-0.2,0.1)$. Shaded and crossed-line regions represent schematic doping regions of AFMM and coexisting AFMM+SC states, respectively. $\langle m\rangle$ represents the staggered magnetization value (vertical axis on the left) and $P_{d}^{a v e}$ ( axis on the right) for SC pairing amplitude (see text for their definition). The results are obtained on the lattice of size $12 \times 12$.

doped holes by taking out the unpaired spin from $\left|\Psi_{1}\right\rangle$. The lowest energy state turns out to be the one with zero total momentum and $S_{z}=0$. Namely, the TWF for two holes with momenta $\mathbf{q}$ and $-\mathbf{q}$ is

$$
\left|\Psi_{2}\right\rangle=P_{d}\left[\sum_{\mathbf{k}}{ }^{\prime}\left(A_{\mathbf{k}} a_{\mathbf{k} \uparrow}^{\dagger} a_{-\mathbf{k} \downarrow}^{\dagger}+B_{\mathbf{k}} b_{\mathbf{k} \uparrow}^{\dagger} b_{-\mathbf{k} \downarrow}^{\dagger}\right)\right]^{\left(N_{s} / 2\right)-1}|0\rangle .
$$

Note that momentum $\mathbf{q}$ is not included in the summation. It is most surprising to find that, although $\left|\Psi_{2}\right\rangle$ has zero total momentum irrespective of $\mathbf{q}$, its energy varies with the missing momentum or the hole momentum q. Same type of TWF for four holes with momenta $\pm \mathbf{q}$ and $\pm \mathbf{q}^{\prime}$ both excluded from the summation can be constructed. The energy dispersion for two holes doped into the halffilled state is also almost identical to that of a single hole and the minimum is at $\mathbf{Q} / 2^{8}$. The MDF for this state has both dips at $\mathbf{Q} / 2$ and $-\mathbf{Q} / 2$. This is in $\operatorname{good}$ agreement with the exact result for the $t-t^{\prime}-t^{\prime \prime}-J$ model with 2 holes in 32 sites. For 2 electron doped case, the dispersion turns out to be very similar to that of a single doped electron ${ }^{8}$. The state with momentum $(\pi, 0)$ has the lowest energy for two electrons.

Our results reproduce the contrasting behaviors between lightly hole and electron doped [e.g. $\mathrm{Nd}_{2-x} \mathrm{Ce}_{x} \mathrm{CuO} \mathrm{O}_{2}$ (NCCO)] high $T_{c}$ cuprates revealed by high-resolution ARPES 2.15. While a small hole patch is observed to be at the center in the nodal direction of the BZ $[$ i.e. $(\mathbf{Q} / 2)]$ in hole doped systems, small electron patches centered at $(\pi, 0)$ and $(0, \pi)$ are observed in lightly doped NCCO.

Focusing on the hole doped case, we found that this type of TWF with even number of holes have AF LRO but very little superconducting pairing correlations- namely, $P_{d}^{a v e}$, the averaged value of the 
long-range part of $d$-wave pair-pair correlation function, $P_{d}(\mathbf{r})=\left(N_{s}\right)^{-1} \sum_{i}\left\langle\Delta_{i}^{\dagger} \Delta_{i+\mathbf{r}}\right\rangle$ where $\Delta_{i}=c_{i, \uparrow}\left(c_{i+\mathbf{x}, \downarrow}+\right.$ $\left.c_{i-\mathbf{x}, \downarrow}-c_{i+\mathbf{y}, \downarrow}-c_{i-\mathbf{y}, \downarrow}\right)$, for $|\mathbf{r}|>2$ in the $12 \times 12$ lattice is only about $10^{-4}$ ( $\mathbf{x}$ and $\mathbf{y}$ are unite vectors in the $x$ and $y$ directions, respectively). The tiny pairing strength is also indicated by the very small value of $n . n$. hole-hole correlation function, $\left(N_{s}\right)^{-1} \sum_{i}\left\langle\left(1-n_{i}\right)\left(1-n_{i+\mathbf{r}}\right)\right\rangle$ with $n_{i}$ the electron number at site $i$, as shown in Fig. 22(d). This state with large staggered magnetization, small Fermi patch, but no SC pairing behaves like an AFMM [see Fig. [1 and Fig. 2(a) and (d) $\frac{8}{3}$. Note that this state has been found in the inner planes of the $\mathrm{Hg}$-based 5-layered cuprates $\mathrm{HgBa}_{2} \mathrm{Ca}_{4} \mathrm{Cu}_{5} \mathrm{O}_{12+\delta} \frac{10}{}$.

As more holes are doped into the system, the ground state may be switched from AFMM to the one described by WF,
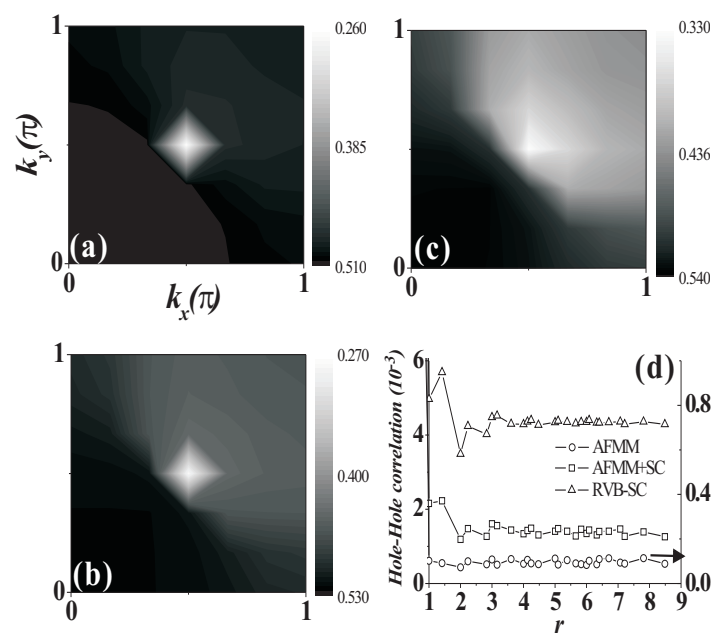

$$
\left|N_{e}\right\rangle_{A F M M+S C}=P_{d}\left[\sum_{\mathbf{k}[\mathbf{S B Z}]}\left(A_{\mathbf{k}} a_{\mathbf{k} \uparrow}^{\dagger} a_{-\mathbf{k} \downarrow}^{\dagger}+B_{\mathbf{k}} b_{\mathbf{k} \uparrow}^{\dagger} b_{-\mathbf{k} \downarrow}^{\dagger}\right)\right]^{N_{e} / 2}|0\rangle,
$$

where $N_{e}$ is now the number of electrons and $\pm \xi_{\mathbf{k}} \equiv \pm \xi_{\mathbf{k}, 1}= \pm\left[\left(\cos \mathrm{k}_{x}+\cos \mathrm{k}_{y}\right)^{2}+\left(m_{s v}\right)^{2}\right]^{\frac{1}{2}}-\mu_{v}-$ $t_{v}^{\prime} \cos \mathrm{k}_{x} \cos \mathrm{k}_{y}-t_{v}^{\prime \prime}\left(\cos 2 \mathrm{k}_{x}+\cos 2 \mathrm{k}_{y}\right)$ containing 3 new variational parameters $\mu_{v}$, for chemical potential, and $\left(t_{v}^{\prime}, t_{v}^{\prime \prime}\right)$, for long range hoppings. This state, which we denote as $\mathrm{AFMM}+\mathrm{SC}$, has stronger superconductivity than that of AFMM state $\left[P_{d}^{a v e}\right.$ is about $5 \times 10^{-3}$, see Fig. 11 and finite correlation between n.n. holes, see Fig. [2(d)] and more extended MDF [Fig. 2(b)]. SC and AF ordered states are uniformly mixed or coexisted in the $\mathrm{AFMM}+\mathrm{SC}$.

Further increasing the number of doped holes in the system, the ground state becomes the RVB-SC state, namely,

$$
\left|N_{e}\right\rangle \equiv P_{d}\left|N_{e}\right\rangle_{0}=P_{d}\left(\sum_{\mathbf{k}} a_{\mathbf{k}} c_{\mathbf{k} \uparrow}^{\dagger} c_{-\mathbf{k} \downarrow}^{\dagger}\right)^{N_{e} / 2}|0\rangle,(4)
$$

where $a_{\mathbf{k}}=v_{\mathbf{k}} / u_{\mathbf{k}}=\left(E_{\mathbf{k}}-\epsilon_{\mathbf{k}}\right) / \Delta_{\mathbf{k}}$ with $v_{\mathbf{k}}$ and $u_{\mathbf{k}} d$ wave SC coherent factors. Here, $\epsilon_{\mathbf{k}}=\xi_{\mathbf{k}, 1}\left(m_{s v}=0\right)$ in the unit of n.n. hopping amplitude. As shown in Fig. 1 and Fig. 2(c) and (d), this state shows even stronger SC pairing amplitude and has a large Fermi surface ${ }^{9}$.

The evolution of states, from AF LRO, AFMM, $\mathrm{AFMM}+\mathrm{SC}$ to RVB-SC, we described above for ideal 2-dimensional system have been realized recently in multilayer system which is considered to have perfectly flat $\mathrm{CuO} \mathrm{O}_{2}$ planes 10 . Indeed, the ground state phase diagram, Fig. [1 as determined for proper set of parameters $\left(t^{\prime}, t^{\prime \prime}\right) / t$ is similar to the one by experiments.

Properties of the quasi-hole and -particle excitations. We then discuss our attempt to apply the TWF approach to study the properties of quasi-hole and -particle excitations of the RVB-SC ground state. We have calculated the quasi-hole excitation spectrum of the SC state and deduced the normal-state Fermi surface. Without any disorder in the system, we also examined

FIG. 2: Plot of MDF's for $12 \times 12$ sites obtained by (a) $\operatorname{AFMM}(x=0.014)$, (b) AFMM+SC $(x=0.042)$, and (c) RVB$\mathrm{SC}(x=0.069)$ states with the same parameters as that in obtaining results shown in Fig.1. (d) The hole-hole correlation functions for different WF's, as denoted by different symbols. Vertical axis on the right (for AFMM) is with a finer scale than that on the left.

the effects of strong correlation exactly by investigating the SW's of the uniform SC state on finite square lattices 16 .

We consider the quasi-particle excitation defined by

$$
\left|N_{e}+1\right\rangle \equiv P_{d} c_{\mathbf{k} \sigma}^{\dagger}\left|N_{e}\right\rangle_{0}
$$

and the quasi-hole one,

$$
\left|N_{e}-1\right\rangle \equiv P_{d} c_{-\mathbf{k}-\sigma}^{\dagger}\left|N_{e}-2\right\rangle_{0} .
$$

By applying the $t-t^{\prime}-t^{\prime \prime}-J$ Hamiltonian to $\left|N_{e}-1\right\rangle$, we calculate its excitation energy for each momentum and then fit the results by the excitation dispersion, $E_{\mathbf{k}}=$ $\left(\epsilon_{\mathbf{k}}^{2}+\Delta_{\mathbf{k}}^{2}\right)^{1 / 2}$ with $\epsilon_{\mathbf{k}}=\xi_{\mathbf{k}, 1}\left(m_{s v}=0\right)$. The Fermi surface is then determined by the zero energy contour of $\epsilon_{\mathbf{k}}$. The doping dependence of the hole density, deduced from the area of the determined Fermi surface is shown in Fig. 31(a). With different sets of $\left(t^{\prime}, t^{\prime \prime}\right) / t$ which are believed to be material dependent- for example, in Fig. 3(a), $(-0.3,0.2)$ for $\mathrm{Ca}_{2-x} \mathrm{Na}_{x} \mathrm{CuO}_{2} \mathrm{Cl}_{2}$ and $(-0.1,0.05)$ for $\mathrm{La}_{2-x} \mathrm{Sr}_{x} \mathrm{CuO} \mathrm{O}_{2}{ }^{17.18 .19}$, the results are consistent with that determined by ARPES. We may also examine the Fermi surface position along the nodal direction, i.e. along $(0,0)-(\pi, \pi)$, at each hole doping density [see Fig. 3(b)]. As can be seen clearly, the Fermi surface position moves more prominently for the case with larger $\left(t^{\prime}, t^{\prime \prime}\right) / t$ values, again consistent with the experimental observation (see also Ref ${ }^{20}$ for Bi-based bilayer cuprates).

Let us then move to calculate the SW for adding (and 


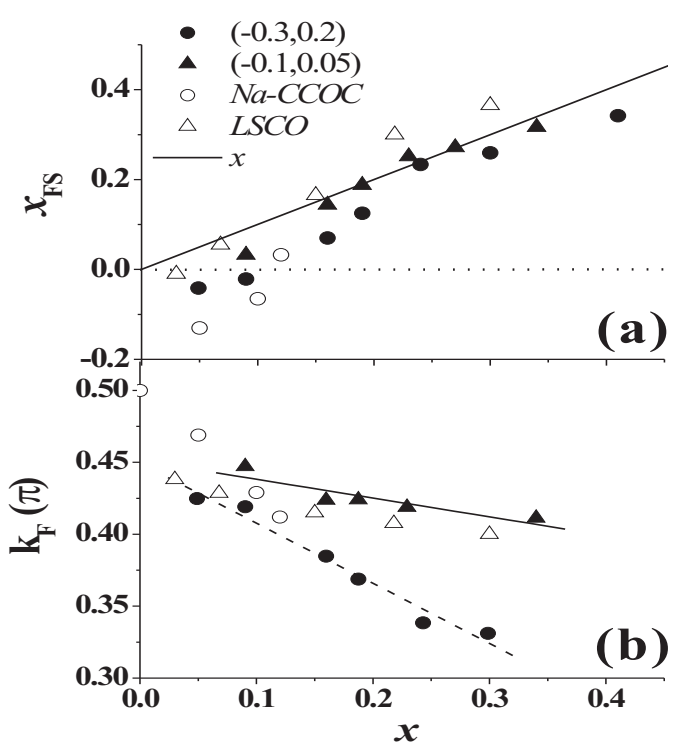

FIG. 3: Doping dependence of (a) the hole density $x_{F S}$ and (b) the $\mathbf{k}_{F}$ position at the nodal point obtained from the fitted quasi-hole dispersion. (See text for the detail.) Solid line in (a) represents the relation $x_{\mathbf{F S}}=x$. Solid circles and triangles are numerical results for $\left(t^{\prime}, t^{\prime \prime}\right) / t=(-0.3,0.2)$ and $(-$ $0.1,0.05)$, respectively. Dashed and solid lines in (b) are linear fitting to these two sets of results. Empty symbols represent experimental data extracted from ARPES, with $\mathrm{Na}-\mathrm{CCOC}$ for $\mathrm{Ca}_{2-x} \mathrm{Na}_{x} \mathrm{CuO}_{2} \mathrm{Cl}_{2}$ and $\mathrm{LSCO}$ for $\mathrm{La}_{2-x} \mathrm{Sr}_{x} \mathrm{CuO}_{2}{ }^{18}$.

removing) one electron defined by

$$
Z_{\mathbf{k} \sigma}^{+(-)}=\frac{\left|\left\langle N_{e}+(-) 1\left|c_{\mathbf{k} \sigma}^{\dagger}\left(c_{\mathbf{k} \sigma}\right)\right| N_{e}\right\rangle\right|^{2}}{\left\langle N_{e} \mid N_{e}\right\rangle\left\langle N_{e}+(-) 1 \mid N_{e}+(-) 1\right\rangle}
$$

Applying identities for projection operator,

$$
\begin{aligned}
{\left[c_{\mathbf{k} \sigma}, P_{d}\right] P_{d} } & =0 ; \\
P_{d} c_{\mathbf{k} \sigma}\left[c_{\mathbf{k}^{\prime} \sigma}^{\dagger}, P_{d}\right] & =P_{d}\left[\frac{1}{N_{s}} \sum_{i} e^{i\left(\mathbf{k}^{\prime}-\mathbf{k}\right) \cdot \mathbf{R}_{i \sigma}} n_{i,-\sigma}\right] P_{d}(8
\end{aligned}
$$

with $\mathbf{R}_{i \sigma}$ the position vector of the $i$-th spin $\sigma$ in the lattice of size $N_{s}$ and $n_{i \sigma}=c_{i \sigma}^{\dagger} c_{i \sigma}$, we can relate $Z_{\mathbf{k} \sigma}^{+}$ exactly to the hole doping and MDF as

$$
Z_{\mathbf{k} \sigma}^{+}=\frac{1+x}{2}-n_{\mathbf{k} \sigma}
$$

21.22 .23 .

On the other hand, reminiscent of what have been argued previously by analytic approach ${ }^{24}$, we recognize that the strong correlation effects becomes apparent only in $Z_{\mathbf{k} \sigma}^{-}$at low doping. The effects due to strong correlation are examined by comparing the coherent SW averaged over all momenta, i.e. $Z_{\text {ave }}^{-} \equiv \sum_{\mathbf{k}} Z_{\mathbf{k} \sigma}^{-} / N_{s}$, and the incoherent part defined by the relation

$$
n_{\text {ave }}^{\text {inc }} \equiv n_{\text {ave }}-Z_{\text {ave }}^{-}
$$

obtained by exact treatment of the projection and by using renormalized mean-field theory $(\mathrm{RMFT})^{25}$. Here $n_{\text {ave }} \equiv \sum_{\mathbf{k}} n_{\mathbf{k} \sigma} / N_{s}$ is the average MDF which should always be equal to the electron density of the system.

The exact results for the $12 \times 12$ lattice and that by RMFT are shown in Fig 4 (a). The coherent part of $Z_{\mathbf{k} \sigma}^{-}$by RMFT is $g_{t} v_{\mathbf{k}}^{2}$ with renormalization factor $g_{t}=2 x /(1+x)$. Completing the momentum sum for the coherence factor, the average result is $x(1-x) /(1+x)$ and, thus, $n_{\text {ave }}^{\text {inc }}=\left[(1-x)^{2}\right] / 2(1+x)^{26}$. The RMFT results are plotted in Fig 4 (a) (dashed and dotted lines) in comparison with the exact ones. As is shown there, while the numerical $n_{\text {ave }}$ is indeed equal to the electron density, the exact incoherent SW for removing an electron is less than the RMFT result. The difference becomes more significant as hole doping level is reduced. Interestingly, this behavior is independent of the $\left(t^{\prime}, t^{\prime \prime}\right) / t$ values [represented by solid and empty symbols in Fig [4(a)] which correspond to very different doping dependence of the Fermi surface shape and also the density of states. By contrast, the average values of $Z_{\mathbf{k} \sigma}^{+}$calculated exactly (not shown) and by RMFT are identical due to Eq.(9).

As a brief digression to electron doped case, it is straightforward to show that, applying the hole-particle transformation to Eq. (9), SW for removing an electron in electron doped system satisfies the relation $Z_{\mathbf{k} \sigma}^{-}=n_{\mathbf{k} \sigma}-$ $(1-x) / 2$ rigorously. The doping dependences of $Z_{\text {ave }}^{-}$ and $n_{\text {ave }}$ are shown in Fig. 4(b). The numerical results is exactly equal to RMFT ones indicating that there is no incoherent part of SW for removing an electron in the electron doped system.

To make a comparison with tunneling experiments, we then concentrate on the SW's as a function of the excitedstate energy. To reduce the effect of finite size, we define the sum of $Z_{\mathbf{k} \sigma}^{ \pm} / N$, over momentum $\mathbf{k}$ which has energy within $E-\Delta E / 2$ and $E+\Delta E / 2$, as $g(E)$ which could be viewed, approximately, as proportional to the conductance at low energy $E$. We plot $g(E)$ in Fig. 5] up to about the energy where peaks appear for lattices of size $12 \times 12$ with $\Delta E / t=0.3$ for various dopings. (The energy interval we chose is to reduce the effects due to lattice size and also to include about the same number of momentum points below and above zero energy.) To make sure our treatment is correct, we have also applied the same analysis to the $d$-wave BCS ( $d$-BCS) state and found the ideal BCS result is hardly distorted by the finite size. Therefore, with the reasonable finite-size dependence, we obtain indeed the $V$-shape $d$-wave gap near zero energy. The width between peak positions is also roughly equal to two times of the gap value deduced from the excitation energy. Looking at the results closely, while $g(E)$ may indeed be about the same at the opposite sides in the very vicinity of zero energy as suggested in Ref ${ }^{23}$, $g(E)$ for removing an electron is always larger than that for adding an electron at higher energy near that of the peak. With decreased doping, the ratio of $g(E)$ at negative and positive energies enhances quite dramatically, e.g. from $x=0.125$ to $0.056, g(-\Delta) / g(\Delta)$ at the corre- 


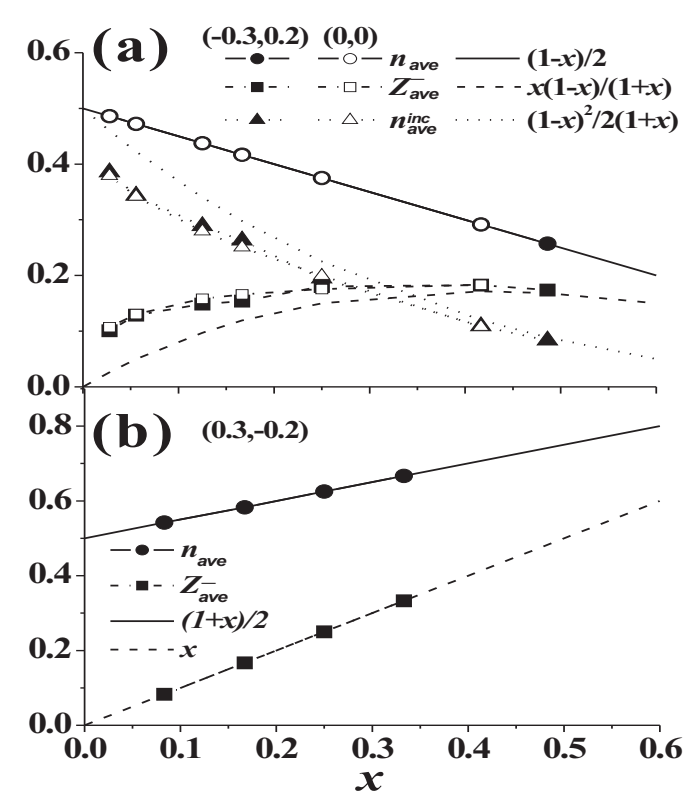

FIG. 4: The doping dependence of average SW and MDF of RVB-SC state in hole doped (a) and electron doped (b) systems, obtained numerically for the $12 \times 12$ lattice and by RMFT. Numerical and expected $n_{\text {ave }}$ are denoted by the circles and the solid line, respectively. The squares (triangles), connected by dashed (dotted) line as the guide for the eye, are for exact results of $Z_{\text {ave }}^{-}\left[n_{\text {ave }}^{\text {inc }}\right.$ only for hole doped case, extracted using Eq. (10) with $n_{\text {ave }}$ calculated numerically]. The dashed and dotted lines without data points represent results by RMFT. All solid symbols in (a) are results for $\left(t^{\prime}, t^{\prime \prime}\right) / t=(-$ $0.3,0.2)$ and empty ones for $(0,0)$. Results in (b) are all for $\left(t^{\prime}, t^{\prime \prime}\right) / t=(0.3,-0.2)$.

sponding peak energy $\Delta$ (in units of $t$ ) increases from 1.96 to 2.73. Similar behaviors are found for the case with vanishing $\left(t^{\prime}, t^{\prime \prime}\right) / t$, as shown in the inset of Fig. 5 In contrast to this, for the $d$-BCS case in the same finite lattices there is almost no change of the ratio within the gap ${ }^{16}$. The numerical results thus tells us features due to strong correlation, i.e. the particle-hole asymmetry of average conductance exists even within the gap region and gets enhanced with underdoping, which are not yet fully explored in the tunneling experiment.

We can also see, from Fig. [5] the correlation between heights of the spectral weight peak and the gap size as doping level is varied. For the two doping level shown there, the peak height scales with the pairing amplitude but anti-correlates with the gap size. This is in clear contrast to the BCS case in which the peak height, proportional to the SC coherence, scales with the gap size as more holes doped into the system. Our result agrees qualitatively with what has been extracted from STS experiments ${ }^{4}$.

To summarize, we discussed $d$-RVB based TWF's de- scribing the ground states of the $t$ - $J$-type models at different hole doping levels. The AFMM, AFMM+SC

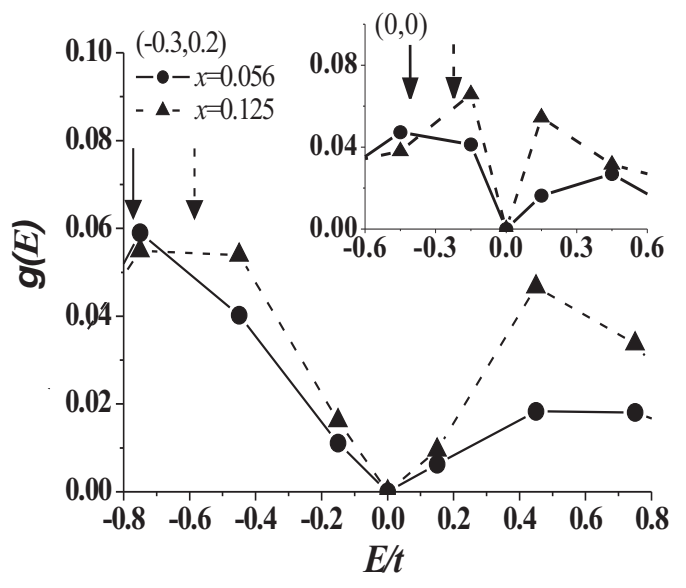

FIG. 5: $g(E)$ for RVB-SC state versus excitation energy $E$ for 2 different dopings. Results shown here are for $\left(t^{\prime}, t^{\prime \prime}\right) / t=(-$ $0.3,0.2)$ and lattice size $12 \times 12$. The associated excitation gap positions are marked by arrows (see text). Inset: Same plots for $\left(t^{\prime}, t^{\prime \prime}\right) / t=(0,0)$. Results for two doping concentration are denoted by the same symbols as that in the main figure.

states have been observed recently in multilayer cuprates. We have also determined the doping dependence of both the Fermi surface area and Fermi momentum along the nodal direction using the fitted quasi-hole dispersion. With proper parameters for different real materials, the results are consistent with the same quantities observed in various high $T_{c}$ cuprates. By studying SW's of the quasi-hole and -particle excitations of the SC state, we found, even without any disorder, features qualitatively consistent with what has been seen in tunneling experiments. An exact relation is obtained for the doping dependence of the spectral weight of removing an electron from an electron-doped cuprates. This could be tested by ARPES. There are also other results which have to be confirmed by future experiments.

We acknowledge discussions with Profs. C. T. Shih, Y. C. Chen, M. Ogata, N. Nagaosa, T. Tohyama, S. Ishihara, V.N. Muthukumar, A. Fujimori, Drs. Y. Yanase and N. Fukushima. We are also grateful to Profs. S. H. Pan, H. Takagi, Y. Kitaoka and Drs. T. Hanaguri and H. Mukuda for kindly sharing their results and insights about their experiments. TKL and CMH are supported by the National Science Council in Taiwan with Grant no.94-2112-M-001-003 and 94-2112-M-032-001, respectively. Part of the calculations are performed in the IBM P690 and SMP2 in the Nation Center for Highperformance Computing in Taiwan. 
1 J. Orenstein and A. J. Millis, Science 288, 468 (2000); D. A. Bonn, Nature Physics 2, 159 (2006).

2 A. Damascelli, Z. Hussain, and Z.-X. Shen, Rev. Mod. Phys. 75, 473 (2003); J. C. Campuzano, M. R. Norman, M. Randeria, in Physics of Superconductors, Vol. II, ed. K. H. Bennemann and J. B. Ketterson (Springer, Berlin, 2004), p. 167-273.

3 C. Renner and Ø. Fischer, Phys. Rev. B 51, 9208 (1995); A. Matsuda, S. Sugita and T. Watanabe, Phys. Rev. B 60, 1377 (1999); M. Kugler et al., J. Phys. Chem. Solids 67, 353 (2006).

${ }^{4}$ K. M. Lang et al., Nature 415, 412 (2002); K. McElroy et al., Phys. Rev. Lett. 94, 197005 (2005).

5 A. C. Fang et al, Phys. Rev. Lett. 96, 017007 (2006).

6 T. Hanaguri et al., Nature 430, 1001 (2004); T. Hanaguri, private communication (2006).

7 P. W. Anderson, Science 235, 1196 (1987).

8 T. K. Lee, C.-M. Ho and N. Nagaosa, Phys. Rev. Lett. 90, 067001 (2003).

9 A. Himeda and M. Ogata, Phys. Rev. B 60, R9935 (1999); C. T. Shih et al., Low Temp. Phys. 31, 757 (2005).

10 H. Mukuda et al., Phys. Rev. Lett. 96, 087001 (2006).

11 D. G. Clarke, Phys. Rev. B 48, 7520 (1993).

12 T. K. Lee and C. T. Shih, Phys. Rev. B 55, 5983 (1997).
13 S. Liang, B. Douçot and P. W. Anderson, Phys. Rev. Lett. 61, 365 (1988); A. W. Sandvik, Phys. Rev. B 56, 11678 (1997).

14 T. Tohyama and S. Maekawa, Phys. Rev. B 49, 3596 (1994); R. J. Gooding, K. J. E. Vos and P. W. Leung, Phys. Rev. B 50, 12866 (1994).

15 F. Ronning et al. Phys. Rev. B 67, 165101 (2003) for holedoped systems; N. P. Armitage et al., Phys. Rev. Lett. 88, 257001 (2002), for electron-doped ones.

16 C.-P. Chou, T. K. Lee and C.-M. Ho, Phys. Rev. B (2006) in press, also as cond-mat/0606633

17 E. Pavarini et al., Phys. Rev. Lett. 87, 47003 (2001).

18 T. Yoshida et al., cond-mat/0510608

19 K.-Y. Yang et al., Phys. Rev. B 73, 224513 (2006).

20 K. Tanaka, Ph.D. Thesis (University of Tokyo, 2004); section 5.3.4.

21 S. Yunoki, Phys. Rev. B72 092505 (2005).

22 C. P. Nave et al., Phys. Rev. B 73, 104502 (2006).

23 H.-Y. Yang et al., cond-mat/0604488

24 W. Rantner and X.-G. Wen, Phys. Rev. Lett. 85, 3692 (2000).

25 F. C. Zhang et al., Supercond. Sci. Tecnnol. 1, 36 (1988).

${ }^{26}$ M. Randeria et al., Phys. Rev. Lett. 95, 137001 (2005). 